\title{
¿Qué es la contabilidad social y ambiental? ${ }^{1}$
}

\author{
Analía Amigo Casson ${ }^{2}$ \\ Recibido: 15 de marzo de 2018 \\ Aprobado: 18 de mayo de 2018
}

\section{Clasificación JEL: Q56}

\section{Resumen}

El presente artículo destaca que el concepto de contabilidad social y ambiental tiene dos corrientes, por un lado, aquellos que la reconocen como una aplicación de la teoría contable en la revelación de hechos sociales y ambientales, y por otro, que utiliza el término contabilidad como un sustantivo totalmente disociado de la teoría contable. Para lograr conocer estas dos vertientes del pensamiento, se recurrió al estudio de documentos publicados por un conjunto de "informantes clave" que fueron seleccionados en razón de su currículum. El trabajo corresponde a un ensayo que presenta los resultados obtenidos de la revisión, defendiendo la clasificación antes mencionada.

Palabras clave: contabilidad social y ambiental, teoría contable, informantes clave.

1 "Este trabajo fue reconocido como uno de los mejores en el "III Congreso Latinoamericano de Estudiantes de Contaduría y Administración -CONTAD 2017”. Ese evento fue organizado por la Asociación Latinoamericana de Facultades y Escuelas de Contaduría y Administración -ALAFEC y se desarrolló en la Universidad de Medellín, en mayo de 2017. Su publicación en la revista Activos es parte del reconocimiento; no está disponible en otra fuente digital".

Citar como: Amigo C., A. (2019). ¿Qué es la contabilidad social y ambiental? Revista Activos, 16(30), 127-152. DOI: https://doi.org/10.15332/25005278.5063

2 Contadora pública y auditora. Facultad de Administración y Economía de la Universidad de Santiago de Chile. Correo electrónico: analia.amigo@usach.cl 


\title{
What is social and environmental accounting?
}

\begin{abstract}
This article states that the concept of Social and Environmental Accounting has two currents, on the one hand those that recognize it as an application of Accounting Theory in the revelation of social and environmental facts, and on the other those who use the term accounting as a substantive fully dissociated from Accounting Theory. To get to know these two currents of thought, we resorted to the study of documents published by a group of "key informants" that were selected based on their curriculum. The work corresponds to an essay that presents the results obtained from the review, defending the aforementioned classification.
\end{abstract}

Keywords: Social and environmental accounting, accounting theory, key informants.

\section{Introducción}

La contabilidad a lo largo de la historia se ha visto influenciada por el medio socioeconómico en el que se desarrolla, por lo que esta se ha ido adaptando y actualizando a los cambios que sufre dicho medio. Así, en la medida en que la contabilidad entregue información útil para la toma de decisiones, va cumpliendo un papel fundamental en el desarrollo económico. Esto ha llevado a ampliar la disciplina contable, en donde han ido apareciendo distintas especialidades, siendo una de las últimas la contabilidad social y ambiental.

De esta forma, el objetivo de este ensayo es saber qué es contabilidad social y ambiental de acuerdo a lo que dicen distintos autores elegidos según el criterio de informantes clave, los cuales se seleccionaron según sus currículums y trayectoria académica o profesional, consistentes en ser docentes de universidades y que tuvieran varias publicaciones sobre la materia. 
El objetivo principal es demostrar a través de la revisión realizada, que existen distintas corrientes que conciben de diversas maneras el término contabilidad social y ambiental, existiendo algunos que establecen una extrapolación de los fundamentos de la teoría contable vinculada con el registro de hechos económicos, hacia la información de hechos importantes para la sociedad y para el mantenimiento del medio ambiente; además de otras corrientes que exponen hechos sociales y ambientales a través de diversos criterios que escapan a la teoría contable.

\section{El actual paradigma de la contabilidad}

Hablar de contabilidad no es un tema actual, se remite al pasado, siendo una disciplina con una evolución histórica antiquísima. Así, se ha visto influenciada por el dominio social y económico en el que se desarrolla, en donde ha ido evolucionando de acuerdo a los cambios de dicho dominio. Este progreso demuestra una relación entre contabilidad como tal y el medio en el que se desenvuelve.

Diversos autores han llegado a la conclusión de que una de las características de la contabilidad es que entrega información útil para la toma de decisiones, por lo que afecta directamente a las actividades económicas. Así lo establece Langenderfer (1973), en donde afirma que la contabilidad es un sistema de medida y comunicación que sirve para entregar información económica y social con la cual los usuarios puedan emitir un juicio y tomar decisiones relativas a la optimización de recursos y al cumplimiento de sus objetivos. De la misma manera, Cañibano (1997) considera que la contabilidad es una ciencia de naturaleza económica que tiene como fin elaborar información que cubra las necesidades financieras externas, planificación y control. Así también, Gonzalo (1983) plantea que el objetivo de la contabilidad es describir y predecir el estado y evolución económica de una entidad con el fin de comunicar a los usuarios "una información objetiva, relevante y válida para la toma de sus decisiones" (p. 107). De esta manera, la contabilidad cumple un papel fundamental en el desarrollo económico, la cual ha tenido un rol importantísimo dentro de la historia, en donde, según 
el académico Tua (2012), se pueden ilustrar tres circunstancias históricas: "el Renacimiento, el auge del capitalismo en los siglos XVII y XIX y, en tercer lugar, la acuñación del concepto responsabilidad social y el tránsito al paradigma de utilidad en el siglo XX" (p. 96). Asimismo, conforme a lo reflexionado por Escobar (2013), existen cuatro enfoques históricos: el antiguo, el clásico hasta el siglo XIX, el económico hasta el siglo XX y, al igual que Tua (2012), actualmente el paradigma de utilidad. De la misma forma, García y Niño (2003) establecen que en nuestros días ha surgido este último arquetipo en la contabilidad.

Este paradigma sostiene que "las empresas deben informar a la sociedad aquellas actividades que realizan y las afecta con la finalidad de que exista evidencia para que los usuarios individuales la consideren en la toma de decisiones" (Díaz, 2003, p. 32), además de producir evidencia para que el mercado "utilice esta información en la determinación de los valores adecuados que circulan en ella, es decir, la información medioambiental debería implicar un cambio en la valorización de activos de las empresas" (Díaz, 2003, p. 32). Esto se vincula con la responsabilidad social en la orientación del suministro de información útil para la toma de decisiones, la cual permita "no solo el control, sino también la evaluación de la situación económica y, en especial, la predicción de su comportamiento futuro y, con ello, que haga posible la adopción de decisiones adecuadas" (Tua, 2012, p. 100). En este sentido, las decisiones tomadas por las empresas y cualquier unidad económica, deben estar en armonía con el medio en el que se desenvuelven, teniendo una sana convivencia, en donde deben actuar con libertad siendo consecuentes con las decisiones que tomen y responsables de sus actos sociales y medioambientales.

\section{Las empresas y la responsabilidad social}

Si bien es cierto, el mundo empresarial se ha convertido muchas veces en culpable de problemas tales como la extinción de especies, emisión de humos y gases que contaminan la atmósfera y hacen desaparecer paulatinamente la capa de ozono, derretimiento de hielos, lluvia ácida, desechos tóxicos que 
contaminan las aguas, agotamiento y sobreexplotación de recursos naturales, etc., sin embargo, la preocupación por el medio ambiente ya no es un tema ajeno, involucrando a variadas empresas en la sustentabilidad medioambiental, las cuales han estado adoptando códigos de conducta acorde con los valores ecológicos de la sociedad, "los cuales se traducen en la implementación de sistemas de producción limpios y respetuosos con el medio ambiente, al tiempo que se esfuerzan en comunicar al exterior las medidas medioambientales adoptadas y los logros alcanzados" (Archel y Lizarraga, 2001, p. 130).

Entonces, se reconoce la responsabilidad social que tienen las empresas, es decir, "la actitud de la dirección de las empresas que implica un compromiso de efectuar un cambio cultural generado en la preocupación por el impacto que sus actividades tienen sobre la sociedad y el medio ambiente" (García, 2012, p. 215). En otras palabras, las empresas tienen sus propios objetivos y, para cumplirlos, toman una serie de decisiones, las cuales tienen diversas consecuencias, pues desarrollan sus actividades en un entorno social y ambiental en donde afectan a muchos sectores de este entorno, como al medio ambiente, a la comunidad, a los trabajadores, al Estado, etc., por lo que la responsabilidad social juega un importante rol que, según Robbins y Decenzo (2002), va "más allá de lo que requieren las leyes o la economía” (p. 59), en el cual las empresas resaltan las buenas prácticas y la responsabilidad que tienen por el impacto de esas decisiones.

Es así como la contabilidad ha reaccionado frente a la responsabilidad social, aplicando la partida doble a la "captación, medición y representación de aspectos relacionados con aquel concepto" (Tua, 2012, p. 99). Todo este proceso, ha llevado a una ampliación de la contabilidad como disciplina, pues empieza a incluir a la realidad social. Así, desde hace más de treinta años se reconocen diversas ramas diferenciadas o especialidades contables que, según Tua (1995a), son explicadas como una consecuencia del paradigma de utilidad.

De esta manera, al reconocer la responsabilidad social que tienen las empresas, emergen especialidades contables en donde la más reciente en aparecer es la contabilidad social y ambiental. 


\section{Contabilidad social y ambiental y la teoría contable}

No existe un consenso del concepto teoría contable. Son numerosos los alcances que se le ha dado, lo que se debe al intento de conceptualizar, desarrollar y elevar la disciplina contable. La significancia se ha ido estableciendo de acuerdo a los momentos históricos que ha vivido la sociedad década a década; la que ciertamente, tiene su origen en la inminente necesidad de investigar y de "pensar en la contabilidad desde referentes filosóficos, sociológicos, económicos e históricos” (Alzate, 2011, p. 2), en donde se puede ver vinculada con otras áreas del conocimiento, la que puede nutrirse de otros saberes.

Tua (1995b) cita en su obra Evolución del concepto de contabilidad a través de sus definiciones a Eugene de Fages (1924), quien establece la idea de que la contabilidad es pura y se entiende como un método universal con independencia del campo al que se aplique. Y en esta misma línea cita a Montesinos (1978), quien comenta a autores vinculados a esta teoría, en donde establece que "la contabilidad pura es un proceso aplicable al conocimiento de cualquier cosa que tenga existencia física o metafísica" (p. 173), también que con la contabilidad se pueden observar distintos tipos de fenómenos. Más aún, García (1972) considera que toda estructura circulatoria, abstraída de la realidad, puede ser el objeto de estudio de la contabilidad.

Por otro lado, Tua (1993) define que no es lo mismo un sistema contable que la teoría general contable, o sea, es necesario hacer una distinción entre estos dos conceptos, pues el sistema contable es una aplicación de la teoría contable, la cual "se deduce a partir de la misma" (p. 102). Además, el autor señala que la teoría contable está compuesta de "elementos y conceptos comunes que están presentes en todos los sistemas contables que, de este modo, se convierten en aplicaciones, es decir, manifestaciones extraídas de la misma, de la teoría general" (p. 99).

Desde otro punto de vista, Biondi (1998) clasifica a la teoría contable en dos grandes grupos: la teoría contable doctrinaria y la teoría contable normativa. La primera está basada en la "opinión de los autores y de los 
investigadores, sobre temas generales o determinados" (p. 2), donde se encuentran la escuela anglosajona y la europea continental. Por su parte, la teoría contable normativa "es el sustento de las normas prácticas dictadas por organismos autorizados para ello, sobre temas específicos" (p. 7).

Asimismo, Wirth (1999) clasifica a la teoría contable en dos enfoques doctrinarios: la teoría positiva y la teoría normativa. en donde la primera, se encarga de "describir cosas reales que existen o que se producen en el mundo real" (p. 165) y, en consecuencia, "sus enunciados empíricos deberían ser contrastables con la realidad como en otras ciencias fácticas"(p. 165), en otras palabras, la contabilidad positiva pretende estudiar la realidad tal como es, poder "describir y explicar los efectos del uso de los sistemas contables y en lo posible predecirlos" (Fernández y Geba, 2009, p. 149). Por su lado, la teoría normativa tiene una finalidad pragmática que consiste, según Mattessich (1995), en brindar un servicio de información acerca de los recursos que controla la organización para el logro de sus objetivos y de la forma de medir el desempeño. Por lo que el cumplimiento de esta finalidad pragmática implica para la teoría contable que no se aproxime solamente al estudio de una parte de la realidad física y social, es decir, su aspecto descriptivo, sino que también establezca "hipótesis instrumentales" (Wirth, 1999, p. 166), en donde sus enunciados sean prescriptivos del tipo ABC, los cuales implican la situación que, según Wirth (1999), para lograr A (objetivo de la información), dadas las condiciones de C (datos relevantes del contexto en el que se plantea $\mathrm{A}$ ), haga $\mathrm{B}$ (medio en que debe emplearse).

La teoría contable normativa no se denomina así porque prepara los informes basados en un conjunto de reglas o criterios acordados por organismos de control o la profesión misma, se denomina así porque para preparar cualquier tipo de información se tienen que "establecer reglas tecnológicas que aspiran a relacionar medios y objetivos de la manera más eficiente" (Wirth, 1999, p. 167), las cuales están respaldadas por una teoría.

Por su lado, Fernández y Geba (2009) establecen que la contabilidad es una rama del saber, la que cuenta con un amplio conocimiento doctrinario que, aunque demanda una teoría general del conocimiento contable, "sirve 
de base para la elaboración de modelos, reglas, normativas tecnológicamente controladas (normas contables), sistemas, informes, etc." (p. 93). Entonces, se puede dilucidar que a la teoría general contable la forma dicho conocimiento doctrinario junto con la norma.

Sumado a todos estos pensamientos, Lorenzo, García y Geba (1999) establecen un enfoque conceptual jerárquico, el cual se puede esquematizar de la siguiente forma:

Teoría contable $\rightarrow$ modelos contables $\rightarrow$ normas o reglas específicas $\rightarrow$ sistemas contables concretos $\rightarrow$ informes contables (incluyen estados contables) (p. 150).

Los académicos distinguen en el universo del discurso contable tres subprocesos: el de descripción cualitativa y cuantitativa, el de comunicación, y el de evaluación y control.

Estos componentes, según Fernández y Geba (2009), "forman parte de un proceso denominado proceso contable, que genera conocimiento y que puede dividirse con fines metodológicos, en:

Subproceso de descripción y explicación cualitativa y cuantitativa (medición) de recursos y hechos referidos a un ente u organización y elementos componentes: entes u organizaciones (sujetos del proceso) recursos y hechos a describir, componentes, registros, programas contables, ordenadores, etc.

Subproceso de comunicación entre emisores y destinatarios de la información que surge de 1 y sus elementos componentes: entes emisores, entes a los que se refiere la información, destinatarios, informes, etc. Este proceso incluye el subproceso de regulación y sus elementos componentes: entes reguladores, regulaciones o normas. etc.

Subproceso de evaluación y control de los procesos 1 y 2 y de sus elementos componentes tales como: entes revisores y auditores, los informes que de ellos emanan, cuando se realizan atento las respectivas regulaciones a normas contables aplicadas a los mismos, entre otros (p. 276). 
Estos subprocesos abarcan una serie de elementos del discurso contable, los que ofrecen un lineamiento y clasificación de acuerdo a diferentes características cualitativas que se consideran importantes.

De esta manera, se entiende la contabilidad como un saber dinámico, la cual está en constante movimiento y "desarrolla sus conceptos gracias a los procesos de investigación que solidifican su estructura conceptual" (Mejía, 2014, p. 126). Así, la teoría y la práctica permiten sustentar teóricamente esto, soportado "en los resultados éticos y estratégicos de la práctica profesional" (p. 126), lo que sumado a la responsabilidad social que han ido reconociendo las entidades económicas, ha hecho necesario que se desarrolle el concepto de contabilidad, emergiendo ramas relacionadas con la realidad social y ambiental, como es la contabilidad social y ambiental.

Esta especialidad contable ha sido estudiada, desarrollada y divulgada por distintos autores, en donde algunos la conciben como una extensión de la teoría contable, pues piensan en ella como un devenir histórico de un nuevo modelo contable que surge como resultado de un actuar organizacional responsable con el medio ambiente, la naturaleza, la sociedad y la economía, en razón a la revelación de hechos que afectan a estos últimos, en donde utiliza elementos y conceptos comunes que están presentes en todos los sistemas contables, los cuales son extraídos de la teoría contable.

Sin embargo, hay otros autores que consideran que la contabilidad social y ambiental solo se refiere al hecho de informar los aspectos sociales y ambientales que afectan a la entidad, sin que ella tenga relación directa con los elementos del discurso contable.

\section{La contabilidad social y ambiental según informantes clave}

En este punto, el presente trabajo se centró en saber cuál es la concepción de contabilidad social y ambiental, de acuerdo a lo que dicen los distintos autores elegidos según el criterio de informantes clave, los cuales se seleccionaron según sus currículums y trayectoria académica o profesional. 


\section{Rob Gray (2003)}

"Acerca del desorden, los sistemas y la sustentabilidad: hacia una contabilidad y unas finanzas más sociales y ambientales".

Gray (2003) afirma que la contabilidad social y ambiental constituye el aspecto más importante de la disciplina contable, la cual ofrece una manera de recuperar una contabilidad y unas finanzas productivas y morales que ubican a la supervivencia de la especie en su mismo centro, además de ser un subsistema del sistema de contabilidad tradicional.

De esta manera, reconoce a la contabilidad como un sistema, en el que cada sistema es parte de sistemas mayores y comprende sistemas en sí mismo; en donde se estudia la política, las leyes y la economía y en algún lugar dentro de esos subsistemas reside el estudio de la contabilidad y finanzas. La contabilidad debe ser estudiada en el contexto en donde se desarrolla, no se puede estudiar aisladamente.

También el autor se refiere a la "teorización", la cual define como el medio formal de darle un sentido a la disciplina, refleja los niveles de coherencia en cuanto a la explicación y análisis, en donde ocurre en el nivel de los subelementos de la disciplina. Así, la auditoría puede ofrecer cierta coherencia como consecuencia de su naturaleza formalmente regulada; las finanzas, en su vinculación primaria al método y a las raíces teóricas de la "ciencia normal". En el caso de la contabilidad, no existe un lente teórico particular que sea capaz de explicar todos los aspectos del objeto de estudio. La práctica no surge de ninguna teoría coherente existente.

Además, Gray (2003) señala que la contabilidad tradicional solo considera cuentas que son financieras por naturaleza, que reflejan acontecimientos económicos, que se centralizan en entidades organizacionales y que se orienten a un conjunto de usos bastante limitado. Esta limitación reside en la metateoría de la economía, dentro de la cual puede ubicarse la contabilidad. Entonces sugiere que, si la contabilidad debe restringirse a la economía de la organización, solo aquellos aspectos sociales y ambientales de naturaleza económica resultan relevantes para ella. 
El autor también indica que la contabilidad y las finanzas se ubican en contextos organizacionales. Estos contextos están basados en el capitalismo financiero internacional moderno. El capitalismo se ubica en el contexto de las instituciones humanas y de la experiencia que, a su vez, se encuentra dentro del sistema ambiental y planetario.

La contabilidad social y ambiental relaciona las formas de contabilidad y finanzas no solo con el sistema de capitalismo sino también con las consecuencias del capitalismo.

Finalmente, Gray concluye que la contabilidad social y ambiental (mencionadas como un concepto único), ha ido creciendo rápidamente con el contar de los años, pero nunca se potenciará como debería ser si se haya tan limitada por la agenda y el lenguaje de los negocios, así como también por el marco dentro de la ortodoxia actual de los negocios y la contabilidad.

\section{Carlos Larrinaga González (1997)}

"Consideraciones en torno a la relación entre contabilidad y el medio ambiente".

El autor reflexiona acerca de las investigaciones sobre contabilidad medioambiental ${ }^{3}$, en donde clasifica la literatura en cinco líneas:

1. Paradigma del beneficio verdadero. Propuesta de que se cree una contabilidad social que evalúe en qué medida la empresa está cumpliendo con el contrato social, es decir, el contrato implícito de la empresa con la sociedad, en donde la empresa tiene "autorización para poseer y utilizar recursos naturales y contratar empleados" (Díaz, 2003, p. 38). Se evalúan los costos sociales y se comunica a través de informes financieros, en donde se considera la valorización del medioambiente.

2. Paradigma de la utilidad de la información para la toma de decisiones. Propone la realización de informes en donde se busquen nuevas formas de medir los

3 La contabilidad medioambiental es concebida como sinónimo de la contabilidad social y medioambiental (social y ambiental). 
efectos medioambientales para incorporarlos a la toma de decisiones. De igual modo, informar sobre las actividades de la empresa que afectan a la sociedad, las cuales podrían ser descritas o medidas mediante los estados financieros.

3. Una perspectiva crítica sobre la contabilidad medioambiental. Hay dudas acerca de la capacidad de la contabilidad medioambiental para provocar el cambio deseado, pues no se tiene evidencia concluyente que afirme que exista algún tipo de reacción ante la publicación de tal información. Además, la publicación voluntaria de la información medioambiental no es una medida representativa del resultado de la gestión medioambiental de las empresas, porque podría estar condicionada a la necesidad de legitimación ante un fuerte impacto medioambiental.

4. Información social de la empresa. Es el proceso de comunicar y presentar la información medioambiental de las acciones económicas de las organizaciones, la que debe estar en armonía con sus propios objetivos, para así rendir cuentas sobre su responsabilidad con la sociedad, la cual puede o no ser utilizada.

5. Contabilidad medioambiental para el cambio organizativo. La contabilidad se considera como un influyente mecanismo para la gestión económica y social. Todo lo que se contabiliza puede modelar la perspectiva de los actores de la organización sobre lo que es importante, ayudando a crear una visión de la realidad, basada en las categorías del discurso económico implícito en el modelo contable. Un cambio organizativo puede ayudar a desencadenar un cambio organizativo y social, haciendo visibles fenómenos medioambientales que permanecen ocultos.

Larrinaga establece que la teoría de que la contabilidad puede utilizarse como un instrumento para realizar un cambio en las empresas hacia un camino más sostenible, implica asumir que es una actividad autónoma, la que se puede influir de forma voluntaria, desencadenando el cambio social. Y, además de estar inmersa en procesos de cambio social, es el reflejo mismo de la configuración social, en donde es inútil proponer nuevos modelos de contabilidad medioambiental si no se producen los cambios institucionales necesarios en la organización y su entorno que doten de relevancia a las nuevas prácticas contables. 
En cuanto a la información medioambiental, sostiene que puede jugar un papel activo en la construcción del propio modelo de responsabilidad.

El autor se cuestiona si es apropiado o no utilizar medidas financieras para una contabilidad que trate de contabilizar el medio ambiente, en caso afirmativo, es necesario que se le dé un lenguaje monetario en donde se deben contabilizar valores que no se conocen. Además, sostiene que el hecho de querer contabilizar los eventos del medioambiente es limitado, porque olvidan la evaluación de alternativas que podrían ser más deseables desde el punto de vista social.

Finalmente, Larrinaga sostiene que, aunque la contabilidad pueda jugar un papel en la consecución de la sostenibilidad, es necesario que deje lo que no sabe hacer en manos de otros.

\section{María del Carmen Rodríguez de Ramírez (2003)}

"Contabilidad y responsabilidad social: un camino por recorrer".

La autora señala que la contabilidad social y ambiental tiene su núcleo en ser una disciplina social, en donde abarca sujetos y objetos y sus diversas interrelaciones procurando que la información resulte útil. Toma en cuenta aspectos que se vinculan no solamente con la trascendencia económica sino también con las implicancias sociales y éticas.

En contabilidad social y ambiental existen diversos enfoques que se han venido desarrollando durante la última década, como:

a. Planteamientos ubicados dentro de una posición tradicional que se centran en el mercado y en las organizaciones lucrativas.

b. Planteamientos ubicados dentro de una posición tradicional que, dentro del modelo vigente, buscan modificar ciertos comportamientos sociales que ponen en peligro, incluso, la propia subsistencia de las organizaciones.

c. Planteamientos que consideran a la disciplina como un elemento moralizador cuya función debe propender a una evolución democrática. 
Además, la contabilidad social y ambiental y las finanzas ofrecen una manera de recuperar una contabilidad y unas finanzas productivas y morales que ubican a la supervivencia de la especie en su mismo centro.

Finalmente, Rodríguez plantea que la contabilidad puede promover la responsabilidad social "a través de la estructuración de sistemas de información contable que permitan efectuar un seguimiento adecuado del manejo de los recursos y que proporcionen diversos modelos" (p. 72) para obtener "mediciones para que los distintos sectores interesados puedan efectuar sus controles y tomar decisiones derivadas de ellos" (p. 72).

\section{Carmen Fernández Cuesta (2004)}

"El marco conceptual de la contabilidad ambiental. Una propuesta para el debate".

La autora define contabilidad ambiental como la parte de la contabilidad aplicada cuyo objeto son las relaciones entre una "entidad" y su "medio ambiente", en donde "entidad" son los criterios jurídicos o el ciclo de la vida, mientras que el "medio ambiente" es el entorno natural o vital.

Las posibles combinaciones de estos dos conceptos básicos, permiten establecer al menos tres alternativas, de diversa fundamentación económica y alcance, para el marco conceptual:

1. Cuando se opta por delimitar la entidad a partir de criterios jurídicos y el medio ambiente como entorno natural, el marco conceptual de la contabilidad ambiental se fundamenta en la economía tradicional, para la cual la ciencia económica es independiente de cualquier consideración ambiental, excepto en dos aspectos: la naturaleza como productora de recursos económicos y como receptora de desechos.

2. Si el criterio de delimitación de la entidad se deriva del análisis del ciclo de vida de sus productos y actividades y el medio ambiente se entiende como entorno vital, el marco conceptual se fundamenta en la economía ecológica, para la cual 
el sistema económico es un subsistema del sistema ambiental, acorde con sus normas y en continua interacción con él.

3. Si se opta por combinar los diversos criterios de delimitación de la entidad y del medio ambiente en función de los objetivos que persiga la información contable, el marco conceptual se fundamenta en la economía ambiental (posición intermedia entre la economía tradicional y la economía ecológica), para la cual el sistema económico está relacionado y limitado por el sistema ambiental.

Concluyendo, Fernández afirma que, para poder normalizar a la contabilidad ambiental, se necesita que los contadores alcancen un consenso acerca de la metodología y marco conceptual de esta disciplina, lo cual se puede lograr si: a) se defiende a la contabilidad ambiental como una especialidad de la contabilidad, en donde su objeto de estudio es la relación entre una entidad y su medio ambiente y, b) se establece un marco conceptual flexible y adaptable a la información contable a elaborar.

\section{Paula A. D’onofrio (2006)}

"Las teorías de la contabilidad social y su relación con la responsabilidad social empresarial".

La autora define la contabilidad social como aquella que contempla la información que genera la entidad para reflejar diversos aspectos sociales sobre los que puede impactar su actividad, dentro de la cual se pueden encontrar cuestiones relativas a los empleados, la comunidad social, el medio ambiente $\mathrm{u}$ otras cuestiones éticas.

D’onofrio distingue tres paradigmas dentro de la contabilidad social: el funcionalista o de mercado, el radical y el interpretativo o social. El primero incorpora todas las teorías basadas en la teoría económica neoclásica y la teoría clásica de gestión, en donde los argumentos utilizados para explicar la presentación de información son aquellos relacionados con el mercado. En cuanto al paradigma radical, consiste en líneas de pensamiento con un planteamiento crítico sobre la situación existente, el cual está basado en 
argumentos de tipo teórico crítico. Por su parte, el paradigma interpretativo se basa en argumentos sociales que consideran la existencia de un mundo social con una pluralidad de agentes usuarios de la información social generada por las entidades económicas.

Todos estos paradigmas de la contabilidad social tienen relación con la entrega de información, la cual se interrelaciona con la responsabilidad social, en donde las empresas añaden una dimensión social y ambiental a sus planes y presupuestos para así evaluar sus resultados y establecer mejoras.

\section{Inés García Fronti (2012)}

"Problemas de la contabilidad social y ambiental: algunas reflexiones sobre la necesidad de un abordaje interdisciplinario".

La autora señala que la contabilidad social y ambiental se encuentra dentro del saber contable en donde utiliza el método científico y posee un dominio y objeto propio, indicando también que el contenido social es el elemento más importante de la contabilidad.

García cita a Gray (2000), quien dice que "la contabilidad social es la preparación y la publicación de información sobre las interacciones y actividades de carácter social, medioambiental, vinculadas a los recursos humanos, a la comunidad, a los clientes, entre otras, de una organización y, cuando sea posible, las consecuencias de dichas interacciones y actividades" (p. 3).

García señala que las hipótesis en las que se basa la contabilidad social y ambiental son tres: las empresas tienen responsabilidad social, las empresas deben emitir informes sociales y dichos informes sociales deben ser elaborados por contadores según principios contables. Además, cita a Chapman (1982), quien propone reconocer que la contabilidad no es exclusivamente patrimonial o financiera, sino que también incluye a la contabilidad gerencial, a la contabilidad gubernamental, a la contabilidad económica y a la contabilidad social. 
La contabilidad social y ambiental es interdisciplinaria, porque posee su objeto y su dominio propios, pero utiliza, entre otras, de la administración, la teoría de los stakeholders; de la filosofía, la ética; de la psicología, la teoría de las motivaciones; de la sociología, las teorías vinculadas a las conductas de las organizaciones, y asimismo interactúa con la ética perteneciente a la filosofía, con el derecho, responsabilidad social y sustentabilidad (Almagro, 2004).

Finalmente, concluye que la contabilidad con el correr de los años ha estado avanzando respecto a sus vinculaciones interdisciplinarias, en donde se ha abierto camino a otras disciplinas que enriquezcan y fortalezcan futuras investigaciones.

\section{Fabián Leonardo Quinche Martín (2008)}

"Una evaluación crítica de la contabilidad ambiental empresarial".

El autor plantea que la contabilidad es una disciplina, profesional y académica, que busca el control orgánico del flujo de los recursos sociales, ambientales y naturales, en el relacionamiento de las organizaciones, el hombre y la naturaleza. Asimismo, que la contabilidad puede ser una disciplina constructiva o destructiva en la realidad social y natural, además la contabilidad está inmersa en conflictos sociales, políticos, culturales, ambientales, económicos, etc., por lo que pasa a ser un mecanismo de control de la vida diaria, influyendo en la forma en que vivimos, actuamos y comprendemos.

Quinche establece que la contabilidad ambiental nace ligada a la contabilidad social, sin embargo, el autor señala que son independientes una de otra. Además, considera que la contabilidad ambiental tiene cuatro períodos característicos. El primero sería durante la década de 1970, en la cual surgen reflexiones teóricas de la contabilidad ambiental empresarial. En el segundo período en 1980, se enmarcó la presencia predominante del beneficio verdadero, es decir, un enfoque en busca de los costos ambientales, el desarrollo en la contabilidad de costos/gestión medioambiental. Por su parte, en 1990 se 
amplía la visión hacia la construcción de información social y ambiental de carácter público y que podemos afirmar se viene consolidando hasta nuestros días. Un cuarto período, que recogería la historia total de la contabilidad ambiental, estaría representado por el enfoque de la utilidad para la toma de decisiones, dedicada a los mercados de valores y a los inversores de capital, ya sea como un elemento a tener en cuenta en la valoración de empresas (acciones) o como una forma de trazabilidad (derechos ambientales, etc.).

Quinche determina que la contabilidad es utilizada como un medio de reducir el medio ambiente a términos seguros y manejables para la empresa y que es necesario que esta informe sobre las formas en que ella misma afecta la naturaleza y su entorno, además de reconocer las practicas locales, culturales, étnicas y de género en relación con el medio ambiente. De esta manera, la contabilidad ambiental busca informar la manera en que la entidad económica afecta a la naturaleza y a su entorno, proporcionando datos cualitativos, monetarios y no monetarios de impacto ambiental, además de los esfuerzos por "recuperar, mejorar o conservar el entorno y la naturaleza, de las diversas formas de prevención” (p. 213).

\section{Norma Geba, Liliana Fernández Lorenzo y Marcela Bifaretti (2009)}

\section{"Marco conceptual para la especialidad contable socioambiental".}

Las autoras afirman que una manera de satisfacer la demanda actual de información, se puede lograr complementando la información que ofrece la contabilidad tradicional con la que proporciona la contabilidad socioambiental, mediante la cual es posible utilizar indicadores objetivos y subjetivos, expresados en distintas medidas. Aseguran también que la contabilidad socioambiental es una apertura de la contabilidad social, la cual incluye la dimensión ambiental.

Además, sostienen que la contabilidad socioambiental se aboca al estudio de los impactos (tanto culturales como naturales, directos como indirectos) de 
la actividad de los entes en el todo social y viceversa, en donde esta percepción hace referencia a impactos naturales que caracterizan la dimensión ambiental.

Así también señalan que el estudio de la contabilidad social (y ambiental) en el marco de una teoría general contable, permitirá delimitar su campo de actuación y conocer la aplicación de los medios económico-financieros al desarrollo humano, en donde se contribuye a la satisfacción de información de los usuarios sobre áreas sociales y medioambientales; asimismo permitirá comprobar la posibilidad de desarrollo de los componentes más comunes que hacen al campo de acción de la disciplina contable.

De aplicarse los subprocesos de la teoría contable (descripción cualitativa y cuantitativa, comunicacional y evaluación) a la contabilidad socioambiental, podrá obtenerse conocimiento racional del aspecto referente a la porción de la realidad socioambiental considerada.

En cuanto al objetivo y dimensión de la contabilidad socioambiental, se tiene que el primero es obtener y brindar conocimiento metódico y sistemático de la realidad en su dimensión socioambiental, en cuanto a esta última, se entiende por la porción de la realidad considerada.

\section{Miguel Díaz Inchicaqui (2003)}

\section{"La contabilidad social. Origen y paradigmas".}

Díaz establece que la contabilidad es una ciencia de información empresarial, que a lo largo de la historia "ha buscado siempre satisfacer los requerimientos de sus usuarios internos y externos” (p. 31), procesando recursos básicos, sin embargo, no considera que la empresa es un subsistema social que está obligado a administrar con responsabilidad social los recursos que "conllevan al cumplimiento de las aspiraciones y demandas de la colectividad" (p. 31).

En este sentido, se debe tomar en cuenta la responsabilidad social en la contabilidad, con el fin de poder informar cómo está actuando la entidad 
económica en relación con su entorno socioeconómico y natural. Así, se debe adicionar a la información tradicional, datos de tipo social, ético, medioambiental o ecológico, lo que se denomina contabilidad social.

La responsabilidad social para las empresas supone un aumento en la información, en la que se reflejan "diversos aspectos sociales sobre los que puede impactar su actividad como son cuestiones relativas a los empleados, la comunidad social, el medio ambiente y otras cuestiones éticas" (p. 35).

De esta manera, el autor establece que la contabilidad social es el proceso de comunicar los efectos sociales y medioambientales que se desprenden de las actividades que tiene una entidad económica, lo que implica "extender la responsabilidad social a las organizaciones" (p. 36), lo cual no solo proporciona estados financieros para sus propietarios, pues las empresas "tienen responsabilidades más amplias que hacer dinero para sus accionistas" (p. 36).

Díaz identifica tres características de la contabilidad social (p. 36):

1. Suministrar información para la toma de decisiones sobre políticas y prácticas sociales que adopte la empresa en el nivel estratégico de la organización.

2. Contribuir a determinar si las estrategias y prácticas sociales de la empresa son consistentes con las prioridades sociales.

3. Informar a la colectividad sobre la contribución social de la empresa ya sea en los campos de impacto medioambiental como en los recursos humanos y éticos.

A su vez, reconoce ramas de la contabilidad social, como es la contabilidad medioambiental, la cual es una de las ramificaciones más importantes, en donde el inminente crecimiento del deterioro del medio ambiente es provocado principalmente por las actividades empresariales, por ello se hace preciso que las empresas establezcan sistemas de gestión y políticas medioambientales. 
En la Tabla 1 se presenta un cuadro comparativo en donde se recogen, a modo de resumen, las distintas definiciones realizadas por los autores anteriormente mencionados.

Tabla 1. Definición de contabilidad social y ambiental de acuerdo a informantes clave

\begin{tabular}{|c|c|}
\hline Autor & Definición \\
\hline Rob Gray & $\begin{array}{l}\text { Establece una definición en su obra Current developments } \\
\text { and trends in social and environmental auditing, reporting e } \\
\text { Attestation: a personal perspective (2000), preparación y pu- } \\
\text { blicación de información sobre las interacciones y actividades } \\
\text { de carácter social, medioambiental, vinculadas a los recursos } \\
\text { humanos, a la comunidad, a los clientes, entre otras, de una } \\
\text { organización y, cuando sea posible, las consecuencias de dichas } \\
\text { interacciones y actividades. }\end{array}$ \\
\hline $\begin{array}{l}\text { Carlos Larrinaga } \\
\text { González }\end{array}$ & $\begin{array}{l}\text { Creación de visibilidades que promocionan determinadas con- } \\
\text { figuraciones de las organizaciones y de la sociedad. Inclusión } \\
\text { de elementos hasta ahora excluidos por los esquemas contables } \\
\text { que pueden ilustrar las consecuencias medioambientales de las } \\
\text { empresas. }\end{array}$ \\
\hline $\begin{array}{l}\text { María del Carmen } \\
\text { Rodríguez de } \\
\text { Ramírez }\end{array}$ & $\begin{array}{l}\text { Contribuir a propiciar el aumento de la responsabilidad social } \\
\text { a través de la estructuración de sistemas de información } \\
\text { contable que permitan efectuar un seguimiento adecuado del } \\
\text { manejo de los recursos, y que proporcionen diversos mode- } \\
\text { los que permitan obtener mediciones para que los distintos } \\
\text { sectores interesados puedan efectuar sus controles y tomar } \\
\text { decisiones derivadas de ellos. }\end{array}$ \\
\hline $\begin{array}{l}\text { Carmen Fernández } \\
\text { Cuesta }\end{array}$ & $\begin{array}{l}\text { Parte de la contabilidad aplicada cuyo objeto son las relaciones } \\
\text { entre una "entidad" y su "medio ambiente". }\end{array}$ \\
\hline Paula A. D’Onofrio & $\begin{array}{l}\text { Es aquella que contempla la información que genera la entidad } \\
\text { para reflejar diversos aspectos sociales sobre los que puede } \\
\text { impactar su actividad. }\end{array}$ \\
\hline Inés García Fronti & $\begin{array}{l}\text { No tiene una definición propia. Cita a Gray (2000): } \\
\text { Preparación y publicación de información sobre las inte- } \\
\text { racciones y actividades de carácter social y medioambiental } \\
\text { vinculadas a los recursos humanos, a la comunidad y a los } \\
\text { clientes, entre otras, de una organización y, cuando sea posible, } \\
\text { las consecuencias de dichas interacciones y actividades. }\end{array}$ \\
\hline
\end{tabular}




\begin{tabular}{l|l}
\hline $\begin{array}{l}\text { Fabián Leonardo } \\
\text { Quinche Martín }\end{array}$ & $\begin{array}{l}\text { No establece una definición propia de contabilidad social, } \\
\text { utiliza una genérica para definir contabilidad. }\end{array}$ \\
$\begin{array}{l}\text { Es una disciplina, profesional y académica, que busca el control } \\
\text { orgánico del flujo de los recursos sociales, ambientales y natu- } \\
\text { rales, en el relacionamiento de las organizaciones, el hombre y } \\
\text { la naturaleza. }\end{array}$ \\
$\begin{array}{l}\text { Norma Geba, Liliana } \\
\text { y Marcela Bifaretti }\end{array}$ & $\begin{array}{l}\text { Se aboca al estudio de los impactos (tanto culturales como } \\
\text { naturales, directos como indirectos) de la actividad de los entes } \\
\text { en el todo social y viceversa. }\end{array}$ \\
\hline $\begin{array}{l}\text { Miguel Díaz } \\
\text { Inchicaqui }\end{array}$ & $\begin{array}{l}\text { Proceso de comunicar los efectos sociales y medioambientales } \\
\text { de la actividad económica de las organizaciones a determi- } \\
\text { nados grupos de interés en la sociedad y a la sociedad en su } \\
\text { conjunto. }\end{array}$ \\
\hline
\end{tabular}

Fuente: elaboración propia.

\section{Conclusiones}

La responsabilidad social a la que se ven enfrentadas las entidades económicas hoy por hoy, hace necesaria la elaboración y presentación de la información que está relacionada con el medio en el que se desenvuelven estas, ya sea el medio ambiente, la naturaleza y la sociedad misma. De esto, surge la contabilidad social y ambiental.

La contabilidad social y ambiental se desarrolla como una manera de poder entregar información incorporando una mayor cantidad de aspectos, de modo que pueda satisfacer las necesidades de información y sea útil para la toma de decisiones a nivel interno y externo, mostrando así resultados económicos además del impacto ambiental y social que genera el proceso productivo de las organizaciones.

Sin embargo, luego de la revisión de literatura realizada, es posible constatar la falta de un concepto convencional de contabilidad social y ambiental, existiendo propuestas de distintas naturalezas. A su vez, hay autores que consideran a la contabilidad social y ambiental como una sola disciplina, mientras que otros suponen a la contabilidad social y contabilidad ambiental 
como dos conceptos independientes. Así también, existen investigadores que la contemplan como sinónimo de "contabilidad medioambiental" o "contabilidad socioambiental".

Teniendo en cuenta autores como Gray, Larrinaga, Rodríguez, García, Geba, Fernández y Bifaretti, entre otros, es posible afirmar que la contabilidad social y ambiental es una aplicación de la teoría contable, en razón de la revelación de hechos de importancia social y ambiental, ya sea porque esta revelación afecta la situación patrimonial de la entidad, o porque solamente se considera oportuna su exposición.

Por otro lado, existe un grupo de autores como Fernández, D’onofrio, Quinche y Díaz, que conceptualizan la contabilidad social y ambiental como un ejercicio totalmente ajeno a la teoría contable, siendo utilizado este término como un sustantivo que alude al hecho de contar, sumar o simplemente dar cuenta de aspectos ambientales que afectan o que le afectan a la entidad que realiza tal revelación.

Dada la existencia empírica de estas dos corrientes, se puede concluir que el concepto de contabilidad social y ambiental no es algo transversal y que es argumentativo, dependiendo de quién emite o se aventura a postular su definición o utilización teleológica de esta.

\section{Referencias}

Almagro Vázquez, F. (2004). Medición del desarrollo sustentable, reto de las cuentas nacionales. La experiencia de México en el cálculo del producto interno bruto ecológico. Problemas del Desarrollo, 35(139), 93-119. Recuperado de https:// www.redalyc.org/pdf/118/11825939005.pdf

Alzate Giraldo, J. S. (2011). Apuntes sobre teoría contable: sus avances, obstáculos, tendencias y retos. Adversia, 1(8), 1-9. Recuperado de http://aprendeenlinea. udea.edu.co/revistas/index.php/adversia/article/viewFile/10952/10045 
Archel Domench, P. y Lizarraga Dallo F. (2001). Algunos determinantes de la información medioambiental divulgada por las empresas españolas cotizadas. Revista de Contabilidad: Spanish accounting review, 4(7), 129-154.

Biondi, M. (1998). Reflexiones sobre los objetivos de los estados contables. Contabilidad y Auditoría, 4(7), 11.

Cañibano, L. (1997). Teoría actual de la contabilidad: técnicas analíticas y problemas metodológicos. Madrid, España: Instituto de Contabilidad y Auditoría de Cuentas - ICE.

Díaz Inchicaqui, M. (2003). La Contabilidad Social origen y paradigmas, Quipukamayoc, 10(19), 31-42. DOI: https://doi.org/10.15381/quipu.v10i19.5513

D’Onofrio, P. A. (2006). Las teorías de la contabilidad social y su relación con la responsabilidad social empresaria. El trabajo es una versión de la ponencia presentada y defendida en las sesiones del XV Congreso Internacional de la Academia Europea de Dirección y Economía de la Empresa, Buenos Aires, Argentina, septiembre de 2006.

Escobar, M. E. (2016). Historia de la contabilidad. Apuntes Contables, 18, 155-176.

Fernández Cuesta, C. (2012). El marco conceptual de la contabilidad ambiental. Una propuesta para el debate. Contabilidad y Auditoría, 10(19), 10.

Fernández Lorenzo, L. y Geba N. (2009). Lecturas sobre elementos del discurso contable. Buenos Aires, Argentina: Haber, Ciencias Económicas.

García Fronti, I. (2012). Problemas de la contabilidad social y ambiental: algunas reflexiones sobre la necesidad de un abordaje interdisciplinario. Contaduría Universidad de Antioquia, 1(60), 209-218. Recuperado de http://aprendeenlinea.udea.edu.co/revistas/index.php/cont/article/viewFile/14690/12843

García García, M. (1972). Modernas tendencias metodológicas en contabilidad. Revista Española de Financiación y Contabilidad, 1(1), 23-44.

Geba, N. B., Fernández, L. E., y Bifaretti, M. C. (2010). Marco conceptual para la especialidad contable socioambiental. Actualidad Contable Faces, 13(20), 49-60. Recuperado de https://www.redalyc.org/pdf/257/25715828005.pdf

Gray, R. (2003). Acerca del desorden, los sistemas y la sustentabilidad: hacia una contabilidad y unas finanzas más sociales y ambientales. Contabilidad y Auditoría, 9(17), 26. Recuperado de http://www.ojs.econ.uba.ar/ojs/index. php/Contyaudit/article/viewFile/156/258 
Gray, R. (2000). Current developments and trends in social and environmental auditing, reporting and attestation: a review and comment. International journal of auditing, 4(3), 247-268. DOI: https://doi.org/10.1111/1099-1123.00316

Gonzalo Angulo, J. A. (1983). Memoria de la teoría de la contabilidad (origen, desarrollo y contenido actual del análisis circulatorio). Madrid, España: Instituto de Contabilidad y Auditoría de Cuentas.

Langenderfer, H. Q. (1973). A conceptual famework for financial reporting. Journal of Accountancy (pre-1986), 136(000001), 46.

Larrinaga González, C. (1997). Consideraciones en torno a la relación entre la contabilidad y el medio ambiente. Revista Española de Financiación y Contabilidad, 26(93), 957-991.

Mejía Soto, E. (2014). Biocontabilidad: hacia una definición de una nueva disciplina contable. Lúmina, 15, 106-129.

Julve, V. M. (1978). Formación histórica, corrientes doctrinales y programa de investigación de la contabilidad. Técnica Contable, 30(357), 351-360.

Quinche Martín, F. L. (2008). Una evaluación crítica de la contabilidad ambiental empresarial. Revista Facultad de Ciencias Económicas: Investigación y Reflexión, 16(1), 197-216.

Robbins, S. P. (2002). Fundamentos de administración, 3. a edición. México D. F., México: Pearson Educación.

Rodríguez de Ramírez, M. d. C. (2003). Contabilidad y responsabilidad social: un camino por recorrer. Presentado en el 9. ${ }^{\circ}$ Encuentro Nacional de Investigadores Universitarios del Área Contable, Universidad Nacional de La Plata, Facultad de Ciencias Económicas, Instituto de Estudios Contables, La Plata, Argentina, 4 de julio de 2003. Recuperado de http://www.economicas.uba.ar/wp-content/ uploads/2016/03/Contabilidad_y_responsabilidad_social_un_camino_para_ recorrer.pdf

Tua Pereda, J. (1993). La investigación en contabilidad: una reflexión personal. Técnica Contable, 44, 585-608.

Tua Pereda, J. (1995a). Lecturas de teoría e investigación contable. Medellín, Colombia: Centro Interamericano Jurídico Financiero.

Tua Pereda, J. (1995b). La evolución del concepto de contabilidad a través de sus definiciones. Medellín, Colombia: División Editorial CIJUF. 
Tua Pereda, J. (2012). Contabilidad y desarrollo económico. Contabilidad y Negocios, 7(13).

Wirth, M. C. (1999). Posible contribución de la teoría contable para la determinación del valor de una empresa. Contabilidad y Auditoría, 9, 146-179. Recuperado de http://www.ojs.econ.uba.ar/ojs/index.php/Contyaudit/article/ viewFile/198/340 